\title{
Desarrollo de poliuretanos con capacidad de retención de boro fitodisponible
}

\section{Development of polyurethanes with phytoavailable boron retention capacity}

\author{
Manuel S. Palencia ${ }^{*}$, Natalia Afanasjeva², Erika J. Benavidez ${ }^{3}$. \\ Recibido para publicación: Junio 1 de 2015 - Aceptado para publicación: Noviembre 25 de 2015
}

\begin{abstract}
RESUMEN
En el contexto de la fertilización de cultivos con ácido bórico $\left(\mathrm{H}_{3} \mathrm{BO}_{3}\right)$, la utilización de materiales con capacidad de carga (retención de boro) y descarga (liberación del boro retenido), tienen potenciales aplicaciones en el desarrollo de sistemas de fertilización y en el tratamiento de aguas de riego. En el presente estudio se planteó obtener un material compuesto, basado en poliuretanos (PUs) y N-(4-vinilbencil)$\mathrm{N}$-metil-D-glucamina (VbNMDG), mediante la técnica de redes poliméricas interpenetrantes (RPIs), con potenciales aplicaciones en el desarrollo de sistemas de fertilización de boro. Para ello, diferentes matrices de PU fueron sintetizadas a partir de un isocianato (metilendifenil-isocianato, MDI) y polioles de peso molecular variable (etilenglicol, glicerol, manitol). Estos PUs fueron empleados como matriz soporte de una segunda red polimérica sintetizada a partir de la polimerización por radicales libres del VbNMDG, el cual fue sintetizado mediante sustitución nucleofílica entre el $p$-clorometilestireno (CIME) y la N-metil-D-glucamina (NMDG). La capacidad de retención y liberación de boro se evaluó mediante experimentos tipo batch realizándose la cuantificación del boro remanente se hizo por el método de la azometina-H. Los resultados sugieren que las RPIs preparadas a partir de manitol poseen una mayor capacidad de retención de $\mathrm{H}_{3} \mathrm{BO}_{3}$ respecto a las obtenidas con etilenglicol y glicerol. Las propiedades de retención de las RPIs aumentan con el aumento de la relación $\mathrm{R}-\mathrm{OH} / \mathrm{H}_{3} \mathrm{BO}_{3}$. Se concluyó que RPIs pueden ser obtenidas a partir de PUs y poli(VbNMDG) empleando dioxano como solvente.
\end{abstract}

Palabras clave: Boro fitodisponible, N-metil-D-glucamina, poliuretanos, redes poliméricas interpenetrantes.

\begin{abstract}
For fertilization of crops with boric acid $\left(\mathrm{H}_{3} \mathrm{BO}_{3}\right)$, the use of material with capacity of charge (boron retention) and discharge (boron release) has potential applications in the development of fertilization systems and in the treatment of irrigation water. This study sought to obtain a composite material from polyurethanes (PUs) and $\mathrm{N}$-(4-vinylbencil)$\mathrm{N}$-methyl-D-glucamine (VbNMDG) by using the technique of interpenetrating polymer networks (IPN), with potential applications in the development of boron fertilization systems. To that end, several PUs were synthesized from an isocyanate (methylendiphenyl-isocianate, MDI) and polyols with variable molecular weight (ethylene glycol, glycerol and manitol). PUs were used as a support matrix and a second network was synthesized by free radical polymerization of VbNMDG, which was previously synthesized by nucleophilic substitution between 4-vinylbenzyl chloride and N-methylD-glucamine. Capacity of retention and release of boron were evaluated by batch experiments and the quantification of boron concentration was estimated using azomethine- $\mathrm{H}$ method. Results suggest that IPNs obtained from manitol have a larger retention capacity of $\mathrm{H}_{3} \mathrm{BO}_{3}$ than those obtained from ethylene glycol and glycerol. Retention properties of IPNs increase as the $\mathrm{R}-\mathrm{OH} / \mathrm{H}_{3} \mathrm{BO}_{3}$ ratio increases. The study concluded that IPNs can be obtained from PUs and poli(VbNMDG) using dioxane as solvent.
\end{abstract}

Key words: Phytoavailable boron, interpenetrating polymer networks, N-methyl-D-glucamine, polyurethane.

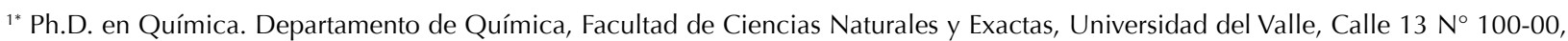
Edif. 320 Ofic. 2063 Sede Melendez. Cali, Colombia; teléfono: (57-2) 3212180, Fax 3212128, correo electrónico: manuel.palencia@ correounivalle.edu.co

2 Ph.D. en Química. Departamento de Química, Facultad de Ciencias Naturales y Exactas, Universidad del Valle, Calle 13 № $100-00$, Edif. 320 Ofic. 2061 Sede Meléndez. Cali, Colombia

${ }^{3}$ Estudiante de Química, Universidad del Valle, Cali, Colombia
} 


\section{INTRODUCCIÓN}

El boro es un elemento químico que se encuentra ampliamente distribuido en la biosfera usualmente en forma de $\mathrm{H}_{3} \mathrm{BO}_{3}$ y otras especies asociadas al borato. En el suelo su concentración y distribución varía ampliamente dependiendo de las propiedades físicas, químicas y microbiológicas presentes (Kot 2015; Abu-Shararet al. 2014; Majidi et al. 2010). Aunque el boro es un elemento esencial para la nutrición y crecimiento de las especies vegetales, en una concentración excesiva produce efectos negativos en las plantas, disminuyendo el rendimiento de la producción de los cultivos y afectando inevitablemente diversos sectores de la economía agraria (Ahmad et al. 2012; Reid 2010).

Desde el punto de vista de la fertilización, un cultivo en el que en el suelo se proporcione una cantidad de boro adecuada para su absorción, será un cultivo con un rendimiento más alto que uno que este en contacto con un suelo que no aporte su requerimiento de boro nutricional. Sin embargo, los límites entre esencialidad y toxicidad del boro para las plantas son muy estrechos y dependen del tipo de cultivo (Handreck 1990; Blevins and Lukaszewski 1998; Navarro y Navarro 2003). Algunos valores de referencia para los cultivos se muestran en la tabla 1. Lo anterior, hace que la dosificación de los contenidos adecuados de boro durante las etapas de fertilización sea un punto crucial para el éxito de las cosechas.

La interacción de cadenas de polioles con ácido bórico ha sido descrita y estudiada en investigaciones previas, encontrándose que la $\mathrm{N}$-metil-D-glucamina (NMDG) es una sustancia con capacidad de acomplejar boro en solución acuosa en función de la acidez del medio (Urbano et al. 2012; Palencia et al. 2014; Vera et al. 2014). Además, la presencia de un grupo nucleofílico (el nitrógeno de la amina) en un extremo permite llevar a cabo la síntesis del vinilbencil-N-metil-D-glucamina (VbNMDG) mediante la reacción con clorometilestireno (CIME), molécula que, a diferencia de la NMDG, es fácilmente polimerizable vía radicales libres. Es posible la obtención de un polímero de alto peso molecular con cadenas laterales de polioles (Palencia et al. 2014).

Por otro lado, las redes poliméricas interpenetrantes (RPIs) son materiales poliméricos que combinan dos redes físicamente unidas de forma no covalente. Esta superposición o entrecruzamiento de redes permite combinar las propiedades de polímeros diferentes en un mismo material. Así, la red puede considerarse constituida por un polímero

Tabla 1. Límites de tolerancia de boro para cultivos (Hu et al. 1996)

\begin{tabular}{lrl}
\hline \multicolumn{1}{c}{ Tolerancia } & \multicolumn{1}{c}{ B $\left(\mathbf{m g ~ L}^{-1}\right)$} & \multicolumn{1}{c}{ Ejemplo de cultivos } \\
\hline Extremadamente sensible & $<0,5$ & Cereza, limón \\
Muy sensible & $0,5-0,75$ & Aguacate, naranja, fresa \\
Sensible & $0,75-1,0$ & Ajo, sésamo, girasol \\
Moderadamente sensible & $1,0-2,0$ & Brócoli, zanahoria, papa, lechuga \\
Moderadamente tolerante & $2,0-4,0$ & Maíz, tabaco \\
Tolerante & $4,0-6,0$ & Alfalfa, tomate \\
Muy tolerante & $6,0-10,0$ & Sorgo, algodón \\
Extremadamente tolerante & $10,0-10,5$ & Espárragos \\
\hline
\end{tabular}


soporte que le otorga, por ejemplo, propiedades mecánicas, y un polímero funcional que suministra alguna propiedad característica asociada con la función que se le desea dar al producto final (en nuestro caso, la capacidad de retener boro, en forma de ácido bórico, desde una solución acuosa) (Ulbricht 2006; Rivas et al. 2011; Lvov and Abdullayev 2013).

En este contexto, al ser los poliuretanos (PUs), polímeros con propiedades ampliamente versátiles y regulables (es decir, poseen una adecuada resistencia mecánica y térmica, se les puede dar forma fácilmente y son resistentes a la biodegradación dependiendo de su síntesis) (Janik and Marzec 2015; Penagos et al. 2015), son candidatos apropiados para actuar como red primaria, o soporte, en la construcción de RPIs. Por consiguiente, una RPI obtenida a partir de PUs y poli(VbNMDG) permitiría obtener un novedoso material con capacidad de retener boro y con potenciales aplicaciones en el desarrollo de dispositivos dosificadores de este micronutriente en condiciones de campo (por ej., sistemas de fertilización en columna o granular) y también podrían ser empleados en el tratamiento de aguas de riego. Estos materiales, en principio, deben cumplir con las siguientes características: bajo costo, adecuadas propiedades mecánicas y capacidad para retener boro bajo determinadas condiciones y liberarlo bajo condiciones de campo.

Por lo anterior, el objetivo del presente estudio fue el desarrollo de nuevos materiales con capacidad de retención y liberación de boro, mediante RPIs construidas a partir de alcoholes polihidroxilados de diferente peso molecular, NMDG y difenilisocianato (MDI).

\section{MATERIALES Y MÉTODOS}

\section{Materiales}

NMDG (Aldrich) y CIME (Aldrich) se emplearon como precursores del VbNMDG. Etilenglicol (Merck), glicerol (Merck) y manitol (Aldrich) fueron usados como polioles en la reacción de uretanización. Metilendifenil-isocianato (MDI, Aldrich) y MDIB (producto comercial, Poliuretano B basado en MDI y catalizadores de reacción) se usaron como fuente de isocianatos. Ciclohexano (Merck), tolueno (Merck) y dioxano (Merck) se evaluaron como solventes de la reacción. La N,N-metilen-bisacrilamida (MBA, Aldrich) se usó como agente entrecruzador y persulfato de amonio (PSA, Aldrich) como iniciador de polimerización. $\mathrm{H}_{3} \mathrm{BO}_{3}$ (Aldrich) se usó como fuente de boro.

\section{Obtención de las matrices de PU}

En primer lugar se llevó a cabo la selección del medio de reacción mediante la síntesis de PUs en diferentes solventes. En cada procedimiento se efectuó la variación del solvente, del isocianato, del alcohol y de la relación isocianato:alcohol. Las relaciones $\mathrm{MDI}: \mathrm{OH}$ analizadas fueron 1:1, 1:2, 1:3.

En primer lugar, se pesaron 2,03 g del isocianato para las distintas matrices de PU, a partir de MDIB y MDI, con y sin solventes; posteriormente, se adicionaron 0,49 $\mathrm{g}$ del respectivo alcohol y se realizó la mezcla en el medio de reacción. En el caso del uso de solventes los reactivos se disolvieron por separado en el solvente respectivo. Después de la mezcla, ésta se dejó en reacción a temperatura ambiente aproximadamente por 15 días hasta reacción completa, la cual se verificó por la formación de un sólido estable. Posteriormente, la mezcla se filtró y se secó a $60{ }^{\circ} \mathrm{C}$ por 8 días hasta peso constante. Los PUs fueron tamizados a través de un tamiz de 90 micrómetros y se caracterizaron por espectroscopia de infrarrojo con transformada de Fourier (FT-IR) usando un espectrofotómetro Shimadzu mediante la técnica de la pastilla de $\mathrm{KBr}$. Además, la composición relativa de $\mathrm{C}, \mathrm{N}$ y O se determinó por análisis elemental.

\section{Síntesis del VbNMDG}

Los precursores que se usaron para la síntesis 
del monómero VbNMDG, fueron NMDG y CIME. Esta se realizó a una razón molar 1:1 de acuerdo con lo descrito por Urbano et al. (2012) y modificado por Palencia et al. (2014). Para llevar a cabo la síntesis se disolvieron 21,9 mmol de NMDG en una mezcla de dioxano y agua desionizada (relación molar 2:1). La solución se adicionó posteriormente a un reactor tipo batch y se calentó por 20 min hasta que la NMDG se disolvió completamente. Después, 3,20 mL (21,9 mmol) de CIME se disolvieron en $10 \mathrm{~mL}$ de dioxano y se adicionaron lentamente al reactor. La reacción permaneció en reflujo con agitación constante durante $5 \mathrm{~h}$. El exceso de CIME se eliminó de la mezcla de la reacción mediante lavado con éter etílico. La síntesis del monómero se verificó mediante un test de polimerización, por FT-IR y análisis elemental.

\section{Formación de las RPIs}

La formación de las RPIs se llevó a cabo mediante la polimerización del VbNMDG en presencia de los PUs sintetizados. Para ello, se hizo reaccionar $0,1 \mathrm{~g}$ de cada uno de los PUs con $2 \mathrm{~mL}$ de la solución de monómero funcionalizado obtenida directamente de la síntesis del VbNMDG. Además, se adicionaron 0,0108 g de MBA y 0,0037 g de PSA. La reacción se llevó en atmosfera de nitrógeno a $75{ }^{\circ} \mathrm{C}$ y el calentamiento se realizó por 4 horas.

\section{Evaluación de la capacidad de retención de boro}

Para los experimentos de retención se utilizó 0,01 g de muestra, se puso en contacto con diferentes cantidades de una solución de $\mathrm{H}_{3} \mathrm{BO}_{3}$ con una concentración $2168 \mathrm{mg} \mathrm{L}^{-1}$. El tiempo de contacto fue de 1 semana para garantizar la completa difusión del $\mathrm{H}_{3} \mathrm{BO}_{3}$ hacia el interior de la matriz polimérica. Las relacione $\mathrm{H}_{3} \mathrm{BO}_{3}$ :$\mathrm{OH}_{\mathrm{PU}}$ usadas fueron 1:5, 1:2, 1:1, 2:1 y 5:1. La mezcla $\mathrm{H}_{3} \mathrm{BO}_{3} / \mathrm{PU}$ se filtró y se lavó con agua destilada. Las muestras fueron puestas en contacto con 30,0 mL de solución de $\mathrm{NaOH}$ a pH 10 para llevarse a cabo la descarga del $\mathrm{H}_{3} \mathrm{BO}_{3}$ retenido el cual fue posteriormente cuantificado por espectrofotometría UV/vis a una longitud de onda de $420 \mathrm{~nm}$ aplicando el Método de la Azometina-H (Roig-Navarro et al. 1996; Sah and Brown 1997).

\section{RESULTADOS Y DISCUSIÓN}

Los resultados en los cuales los materiales obtenidos no fueron considerados apropiados son omitidos por no ser de relevancia.

Con el fin de alcanzar la mayor homogeneidad, el solvente empleado como medio de reacción debe, en principio, ser un excelente solvente tanto para los monómeros como para el polímero formado. El resultado obtenido con los diferentes solventes para el MDI y MDIB, se resume a continuación: ciclohexano (-), ciclohexano/benceno $(-)$, benceno $(+)$, tolueno (+) y dioxano (+). Sólo el dioxano resultó positivo para los diferentes alcoholes.

En términos generales, el carácter hidrofóbico del isocianato contrasta con la naturaleza hidrofílica de los alcoholes. Además, debido a la reactividad de los grupos isocianato con el agua, ácidos carboxílicos y alcoholes, solventes de esta naturaleza no son utilizables. A partir de las pruebas de solubilidad, se concluyó que el solvente más apropiado es el dioxano, el cual es un éter cíclico, inerte frente a los grupos isocianato y alcoholes. Además, es lo suficientemente volátil para su fácil eliminación finalizada la reacción y posee miscibilidad con los alcoholes.

\section{Síntesis de los PUs}

Los PUs obtenidos con glicerol y manitol (MDI-G y MDI-M, respectivamente) en relaciones 1:2 y $1: 3$ isocianato:poliol presentaron una mayor dureza con lo cual se dificultó su trituración y tamizaje, por lo que estás muestras fueron descartadas. En términos generales, tanto el glicerol como el manitol aportan un mayor número de grupos hidroxilo al medio de reacción, por lo cual, el entrecruzamiento de las 
cadenas es mayor manifestándose este hecho en una dureza mayor del material. Aunque esto puede resultar útil para materiales estructurales, la obtención de material particulado es de mayor interés al permitir el empaquetamiento en columnas y una mayor área superficial. Los resultados del análisis de su composición elemental se muestran en la tabla 2 .

La clara apreciación visual de la reacción, permite identificar la ocurrencia de cambios del estado de agregación del sistema, pasándose de una solución a la formación de un sólido compacto.

Un espectro de infrarrojo característico de todos los PUs se muestra en la figura 1. En él se puede apreciar la aparición de nuevas bandas de absorción de los grupos $-\mathrm{OH}$ alrededor de $3500 \mathrm{~cm}-1$ las cuales se encuentran asociadas a la incorporación de los polioles en la estructura polimérica. Así mismo, se identifican bandas de absorción asociadas con la presencia de grupos $-\mathrm{NH}$ y $\mathrm{C}=\mathrm{O}$ provenientes de los grupos uretanos alrededor de los $3100 \mathrm{~cm}^{-1}$ y $1700 \mathrm{~cm}^{-}$ 1 , respectivamente. La presencia de vibraciones en el espectro IR tanto grupos únicos de los alcoholes como de grupos únicos del isocianato indican que efectivamente se obtuvieron los PUs respectivos.
Tabla 2. Análisis elemental de los diferentes PUs.

\begin{tabular}{cccccc}
\hline \multirow{2}{*}{ PUs } & Relación & \multicolumn{4}{c}{ Composición elemental } \\
& -OH:-NCO & \multicolumn{1}{c}{$\mathbf{N} \mathbf{N}$} & $\mathbf{\%} \mathbf{C}$ & $\mathbf{\% H}$ & $\mathbf{\%} \mathbf{~}$ \\
\hline PUE & $1: 1$ & 10,07 & 66,60 & 5,73 & 17,60 \\
& $1: 2$ & 10,18 & 67,63 & 5,58 & 16,61 \\
& $1: 3$ & 10,53 & 69,63 & 5,55 & 14,29 \\
PUG & $1: 1$ & 10,61 & 71,68 & 5,80 & 11,91 \\
& $1: 2$ & 9,77 & 67,41 & 5,49 & 17,33 \\
& $1: 3$ & 9,97 & 69,15 & 5,47 & 15,41 \\
PUM & $1: 1$ & 9,19 & 65,23 & 5,99 & 19,59 \\
& $1: 2$ & 10,35 & 68,65 & 5,73 & 15,27 \\
& $1: 3$ & 11,31 & 71,05 & 5,17 & 12,47 \\
\hline
\end{tabular}

PUE: Poliuretano a partir de metilendifenil-isocianato y etilenglicol PUG: Poliuretano a partir de metilendifenil-isocianato y glicerol PUM: Poliuretano a partir de metilendifenil-isocianato y manitol

\section{Síntesis de la $\mathrm{N}$-(4-vinilbencil)-N-metil-D- glucamina (VbNMDG)}

La síntesis del VbNMDG se constató mediante IR. En la figura 2 se muestran los espectros del CME, la NMDG y del VbNMDG. El espectro de la NMDG presenta una banda de tensión característica del enlace $\mathrm{O}-\mathrm{H}$ a $3331 \mathrm{~cm}^{-1}$. Adicionalmente se encuentran dos bandas intensas en 1075 y $1045 \mathrm{~cm}^{-1}$ correspondientes a los enlaces $\mathrm{C}-\mathrm{O}$ y $\mathrm{C}-\mathrm{N}$, respectivamente. Para el espectro del CIME la vibración de tensión

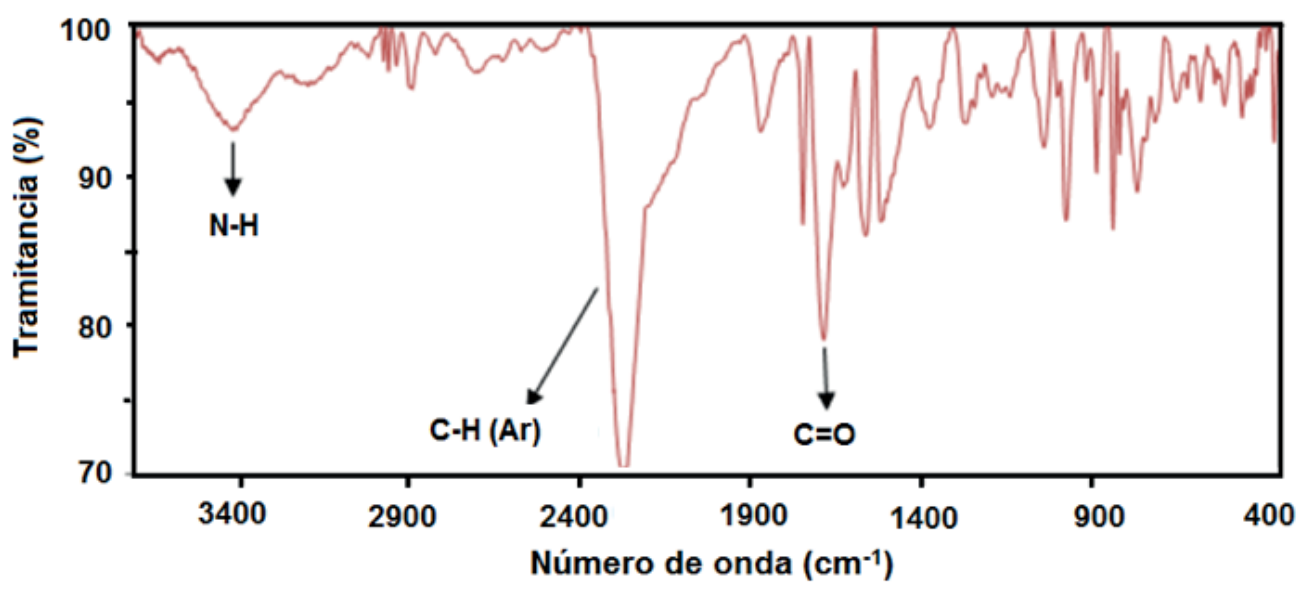

Figura 1. Espectro de infrarrojo característico de los PUs obtenidos. 


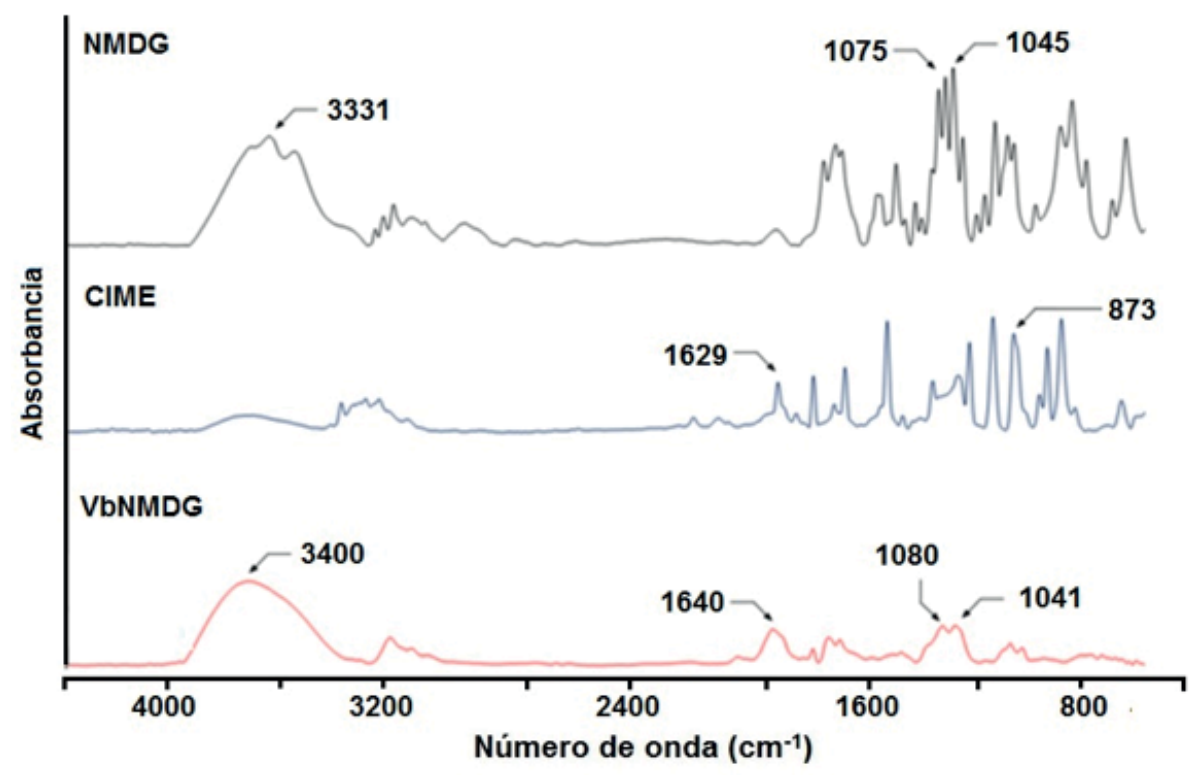

Figura 2. Espectro de infrarrojo característico del vinilbencil-N-metil-D-glucamina y sus precursores: Clorometilestireno (CIME) y N-metil-D-glucamina (NMDG).

del enlace $\mathrm{C}=\mathrm{C}$ se presenta como una banda de moderada intensidad a $1629 \mathrm{~cm}^{-1}$, la banda aguda y fuerte que está presente a $843 \mathrm{~cm}^{-1}$ se atribuye al enlace C-Cl. En el VbNMDG se encuentran tanto las bandas del CIME como de la NMDG, a $3400 \mathrm{~cm}^{-1}$ aparece una banda ancha y fuerte característica del enlace $\mathrm{O}-\mathrm{H}$, en $1640 \mathrm{~cm}^{-1}$ está la banda correspondiente al enlace $\mathrm{C}=\mathrm{C}$, en 1080 y a $1041 \mathrm{~cm}^{-1}$ hay dos bandas correspondientes a la vibración de los enlaces C-O y C-N, respectivamente. Cabe notar que en este último espectro desaparece la banda característica de la vibración del enlace C-Cl, lo cual indica que se llevó a cabo la funcionalización del monómero CIME.

\section{Síntesis de la RPIs a partir de PUs y VbNMDG}

Las RPIs se sintetizaron a partir de los PUs y mediante la reacción de polimerización del VbNMDG. El hinchamiento del PU por efecto del solvente, y la posterior reacción de los solutos disueltos (VbNMDG, PSA y MBA) como resultado del aumento de temperatura. Por consiguiente, mediante la activación térmica del iniciador se forman radicales libres que al reaccionar con el VbNMDG empiezan la reacción de polimerización. A medida que la cadena polimérica crece, la presencia de la MBA enlaza diferentes cadenas de polímero haciendo que tenga lugar la formación de un material entrecruzado en el interior de la matriz de PU. El porcentaje de poli(VbNMDG) incorporado, para las RPIs seleccionadas, se muestra en la tabla 3.

Tabla 3. Porcentaje de poli(VbNMDG) en los diferentes PUs (relación 1:1, MDI:poliol).

\begin{tabular}{cccc}
\hline PUs & $\begin{array}{c}\text { Relación } \\
\text { OH:-NCO }\end{array}$ & $\begin{array}{c}\text { Masa de } \\
\text { muestra }(\mathbf{g})\end{array}$ & $\begin{array}{c}\text { Poli(VbNMDG) } \\
\mathbf{( \% )}\end{array}$ \\
\hline PUE & $1: 1$ & 0,30 & 9,6 \\
PUE & $1: 3$ & 0,09 & 33,8 \\
PUG & $1: 1$ & 0,04 & 12,9 \\
PUM & $1: 1$ & 0,05 & 16,1 \\
\hline
\end{tabular}

PUE: Poliuretano a partir de etilenglicol

PUG: Poliuretano a partir de glicerol

PUM: Poliuretano a partir de manitol 
Capacidad de retención de boro de los PU modificados mediante RPIs

Los PUs seleccionados para estos experimentos fueron PUE (relación 1:3), PUG (relación 1:1) y PUM (relación 1:1). En la figura 3 se pueden observar los milimoles de $\mathrm{H}_{3} \mathrm{BO}_{3}$ por miligramos de RPI. En general, a medida que la cantidad relativa de RPI se incrementa, los contenidos de boro retenido son mayores. Puede notarse que la tendencia no es lineal, siendo esta más pronunciada para mayores contenidos de alcohol y para el PUM. Esto se puede explicar considerando que, a mayores contenidos de alcohol las partículas de PUs son más hidrofílicas y por ende son más susceptible a hincharse (el hinchamiento arrastra una mayor cantidad de solución de $\mathrm{H}_{3} \mathrm{BO}_{3}$ hacia el interior de la matriz polimérica favoreciéndose así su interacción con los grupos hidroxilos). Además, para el manitol, un mayor número de grupos hidroxilo se encuentran disponibles para acomplejar el boro.

La capacidad de los PUs para retener boro se explica mediante la incorporación de las unidades de glucamina las cuales pueden

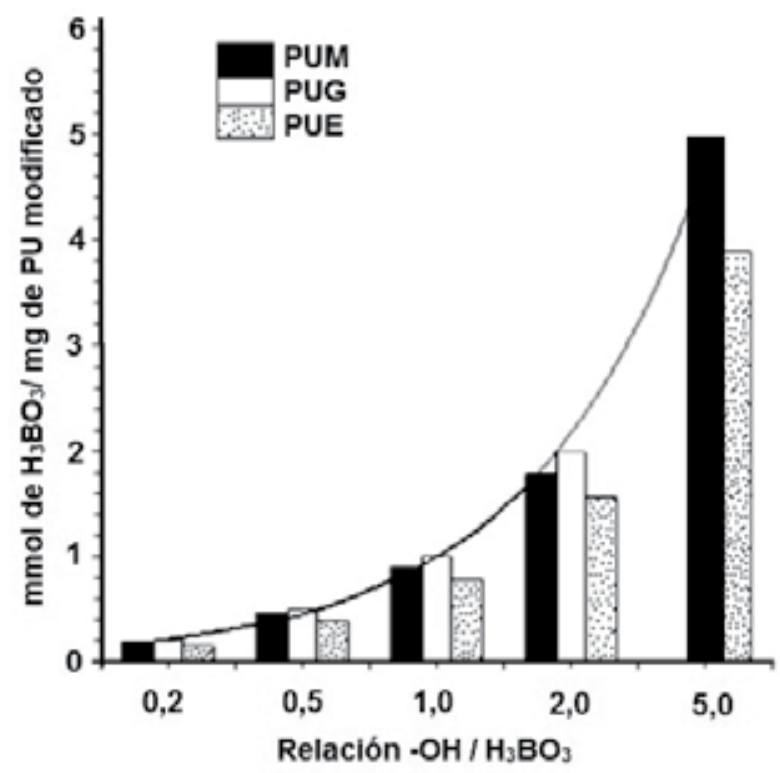

Figura 3. Capacidad de retención de boro de los PUs modificados mediante RPIs. formar complejos tetradentados con el boro como átomo central (Polat et al. 2004). Sin embargo, se debe tomar en consideración que cuando el $\mathrm{pH}>\mathrm{pK}_{\mathrm{a}}$ se favorece la reacción de competición, es decir, el proceso de disociación de $\mathrm{H}_{3} \mathrm{BO}_{3}$ en anión tetrahidroxiborato $\left(\mathrm{B}(\mathrm{OH})_{4}^{-}\right)$. Sin embargo, en los suelos con aptitud agrícola un $\mathrm{pH}>9,2$ corresponde a una situación extrema.

En soluciones acuosas y en condiciones cercanas a la neutralidad, el boro se encuentra en forma de ácido bórico presentando muy poca tendencia a ceder protones. Sin embargo, actúa como ácido de Lewis, por lo que se facilitará la formación del ion hidroborato $\left(\mathrm{B}(\mathrm{OH})_{4}^{-}{ }^{-}\right.$, tal como se muestra a continuación:

$\mathrm{B}(\mathrm{OH})_{3}+2 \mathrm{H}_{2} \mathrm{O} \quad \mathrm{B}(\mathrm{OH})_{4}^{-}+\mathrm{H}_{3} \mathrm{O}^{+} \quad$ [Ec. 1]

La especiación química del ácido bórico en soluciones acuosas se reduce a $\mathrm{B}(\mathrm{OH})_{3}$ y $\mathrm{B}(\mathrm{OH})_{4}$ - debido a que los valores de $\mathrm{pK}_{\mathrm{a}}$ son muy grandes para la segunda y tercera disociación $\left(\mathrm{pK}_{\mathrm{a} 2} \sim 12.4\right.$ y $\left._{\mathrm{pK}} \sim 13.5\right)$ (Navarro y Navarro 2003). Por lo tanto, la capacidad de retención de boro se espera que disminuya a valores de $\mathrm{pH}$ superiores a 9.2 como consecuencia del equilibrio de disociación $\left(\mathrm{pK}_{\mathrm{a}}=9\right.$,2).

\section{CONCLUSIONES}

RPIs pueden ser obtenidas a partir de PUs y poli(VbNMDG) empleando dioxano como solvente. La dureza de los PUs se ve afectada por el contenido del poliol adicionado independientemente de la naturaleza del alcohol (etilenglicol, glicerol o manitol). Las propiedades de retención de las RPIs aumentan con el aumento de la relación $-\mathrm{OH} / \mathrm{H}_{3} \mathrm{BO}_{3}$, siendo mayor a medida que la relación aumenta. Además, los resultados sugieren que las RPIs preparadas a partir de manitol poseen una mayor capacidad de retención de $\mathrm{H}_{3} \mathrm{BO}_{3}$ respecto a las obtenidas con etilenglicol $y$ glicerol. 


\section{AGRADECIMIENTOS}

Los autores agradecen a la Fundación para la Ciencia y Tecnología del Banco de la República por los fondos recibidos en el marco del Proyecto N³518 (C.I. 7001).

\section{REFERENCIAS}

Abu-Sharar, T. Bani, N.and Al-Khader, S. 2014. Boron adsorption-desorption characteristics of irrigated soils in the Jordan Valley. Geoderma Regional, (2), p50-59.

Ahmad, W., Zia, M., Malhi, S. and Niaz, A. 2012. Boron Deficiency in soils and crops: a review. Crop Plant, p77-114.

Blevins, D. and Lukaszewski, K. 1998. Boron in plant structure and function, Annual Reviews Plant Physiology Plant Molecular Biology 49(1):481-500.

Handreck, K. 1990. Methods of assessing boron availability in potting media with special reference to toxicity. Communication in Soil Science Plant Analysis. 21(19-20): 2265-2280.

Hu, H., Brown, P. and Labavitch, J. 1996. Species variability in boron requirements is correlated with cell wall pectin. Journal Experimental Botany 47(2): 227-232.

Janik, H. and Marzec, M. 2015. A review: Fabrication of porous polyurethane scaffolds. Materials Science and Engineering: C. 48:586-591.

Kot, F. 2015. Boron in the Environment. Boron Separation Processes. Elsevier. p 1-33.

Lvov, Y. and Abdullayev, E. 2013. Functional polymer-clay nanotube composites with sustained release of chemical agentss. Progress in Polymer Science,
38(10):1690-1719.

Majidi, A., Rahnemaie, R., Hassani, A. and Malakouti M. 2010. Adsorption and desorption processes of boron in calcareous soils. Chemosphere, 80(7): 733-739.

Navarro, S. y Navarro, G. 2003. Química agrícola: El suelo y los elementos químicos esenciales para la vida vegetal. 2a ed. Mundi-prensa, Barcelona. 487 p.

Palencia, M., Vera, M. and Rivas B. 2014. Modification of ultrafiltration via membranes interpenetrating polymer networks for removal of boron from aqueous solution. Journal Membrane Science 466:192-199.

Palencia, M., Vera, M. and Combatt, E. 2014. Polymer network based in (4-vinylbenzyl)$\mathrm{N}$-metil-D-glucaminesuppourted on microporous polypropylene layers with retention boron capacity. Journal Applied Polymer Science 131(16):40653-40660.

Penagos, D., Restrepo, H. and Palencia, M. 2015. Grafting Polymer Based in Active Polyurethane Matrixes Via Free Radical. Procedia Materials Science 9:491-495.

Polat, H., Vengosh, A., Pankratov, I. and Polat, M. 2004. A new methodology for removal of boron from water by coal and fly ash, Desalination, 164(2):173-188.

Reid, R. 2010. Can we really increase yields by making crop plants tolerant to boron toxicity?.PlantScience, 178(1):9-11.

Rivas, B., Pereira, E., Palencia, M. and Sánchez, J. 2011. Water-soluble functional polymers in conjunction with membranes to remove pollutant ions from aqueous solutions, Progress in Polymer Science, 36(2):294-322. 
Roig-Navarro, A., López, F. and Hernández, F. 1996. Application of azomethine-H method to the determination of boron in workplace atmospheres from ceramic factories, Journal of Analytical Chemistry, 356(1):103-106.

Sah, R. and Brown, P. 1997. Boron determination - a review of analytical methods. Microchemical Journal, 56(3):285-304.

Ulbricht, U. 2006. Advanced functional polymer membranes, Polymer 47(7):2217-262.

Urbano, B. and Rivas, B. 2012. Water-insoluble nanocomposite ion exchange resin based on N-methyl-D-glucamine ligand groups for arsenic removal. Reactive FunctionalPolymer, 72(9):642-649.

Vera, M., Combatt, E. y Palencia, M. 2014. Estudio de la capacidad de retención de boro disponible en suelos mediante membranas funcionales con cadenas de polioles. Temas Agrarios 19 (1):96-105. 